\title{
Cerebral Atrophy
}

National Institute of Neurological Disorders and Stroke (NINDS)

\section{Source}

National Institute of Neurological Disorders and Stroke (NINDS). Cerebral Atrophy

Information Page.

Cerebral atrophy is a common feature of many of the diseases that affect the brain. Atrophy of any tissue means loss of cells. In brain tissue, atrophy describes a loss of neurons and the connections between them. Atrophy can be generalized, which means that all of the brain has shrunk; or it can be focal, affecting only a limited area of the brain and resulting in a decrease of the functions that area of the brain controls. If the cerebral hemispheres (the two lobes of the brain that form the cerebrum) are affected, conscious thought and voluntary processes may be impaired.

Associated Diseases/Disorders: The pattern and rate of progression of cerebral atrophy depends on the disease involved. Diseases that cause cerebral atrophy include:

- stroke and traumatic brain injury

- Alzheimer's disease, Pick's disease, and fronto-temporal dementia

- cerebral palsy, in which lesions (damaged areas) may impair motor coordination

- Huntington's disease, and other hereditary diseases that are associated with genetic mutations

- leukodystrophies, such as Krabbe disease, which destroy the myelin sheath that protects axons

- mitochondrial encephalomyo pathies, such as Kearns-Sayre syndrome, which interfere with the basic functions of neurons

- multiple sclerosis, which causes inflammation, myelin damage, and lesions in cerebral tissue

- infectious diseases, such as encephalitis, neurosyphilis, and AIDS, in which an infectious agent or the inflammatory reaction to it destroys neurons and their axons

Symptoms of cerebral atrophy: Many diseases that cause cerebral atrophy are associated with dementia, seizures, and a group of language disorders called the aphasias. 
- Dementia is characterized by a progressive impairment of memory and intellectual function that is severe enough to interfere with social and work skills. Memory, orientation, abstraction, ability to learn, visual-spatial perception, and higher executive functions such as planning, organizing, and sequencing may also be impaired.

- Seizures can take different forms, appearing as disorientation, repetitive movements, loss of consciousness, or convulsions.

- Aphasias are a group of disorders characterized by disturbances in speaking and understanding language. Receptive aphasia causes impaired comprehension. Expressive aphasia is reflected in odd choices of words, the use of partial phrases, disjointed clauses, and incomplete sentences. 